\title{
Intratumoral heterogeneity of MIB-1 labelling index in gastric gastrointestinal stromal tumor (GIST)
}

\author{
Hiroshi Yasui ${ }^{1,2}$, Eitchi Konishi ${ }^{2}$, Koji Urasaki ${ }^{2}$, Satoru Yasukawa $^{2}$, and Akio Yanagisawa ${ }^{2}$ \\ ${ }^{1}$ Department of Clinical Laboratory, Kyoto First Red Cross Hospital, Kyoto, Japan \\ ${ }^{2}$ Department of Pathology, Kyoto Prefectural University of Medicine, 465 Kajii-cho, Kawaramachi-Hirokoji, Kamigyo-ku, Kyoto 602-0841, \\ Japan
}

\begin{abstract}
Background. The MIB-1 labelling index (LI) is used as a prognostic indicator for gastrointestinal stromal tumors (GISTs). However, whether a biopsy-based LI represents the entire tumor is uncertain, because the $\mathrm{LI}$ is not always homogeneous. In this study, we examined the extent and characteristics of LI heterogeneity in gastric GISTs.

Methods. We analyzed ten c-kit-positive gastric GISTs with diameters exceeding $3 \mathrm{~cm}$, of which six were multilobular and four were unilobular. For MIB-1-immunostained sections, continuous digital images were obtained through the maximum diameter of the lobules. We obtained LIs for images by carrying out computer-assisted image analysis, and calculated the means and standard deviations (SDs) of the LIs for the lobules. For each lobule, intralobular heterogeneity was evaluated on the basis of the SD. For multilobular tumors, interlobular heterogeneity was assessed on the basis of the mean LI difference between lobules.

Results. The SDs, which ranged from $0.8 \%$ to $9.8 \%$, indicated intralobular heterogeneity. Moreover, considerable interlobular heterogeneity was noted in five $(83 \%)$ of the six multilobular GISTs, in which the maximum interlobular mean LI difference ranged from $9.6 \%$ to $27.2 \%$. Notably, although the high maximum mean LI (14.1\%-32.3\%) showed that these five GISTs were high-grade tumors, they also contained at least one lobule showing a low-grade mean LI value $(2.7 \%-$ $5.1 \%$ ).

Conclusion. Gastric GISTs often show intralobular or interlobular MIB-1 LI heterogeneity. In multilobular GISTs, multiple biopsy samples may be required for the accurate evaluation of tumor grade.
\end{abstract}

Key words Ki-67 (MIB-1) · GIST · Intratumoral heterogeneity

\section{Introduction}

Predicting the behavior of gastrointestinal stromal tumors (GISTs) is problematic because of their various degrees of malignancy and their presentation, which ranges from innocent nodules to metastasizing sarcomatous tumors. Good prognostic indicators would help in the selection of the optimal treatment; for this purpose, the mitotic index (MI) and tumor size have been the "gold standard" in clinical pathology [1-3]. On the other hand, the MIB-1 labelling index (LI), another proliferation marker, is a powerful prognostic indicator in carcinomas of various organs and soft-tissue tumors, including GISTs [4-10]. Some investigators have suggested that the prognostic value of the LI may be superior to that of other prognostic indicators, partly due to its low interobserver variability [5-7].

For the assessment of proliferative activity using the LI or MI, the tumor area to be selected should be one where MIB-1-positive cells or mitotic cells are the most densely distributed. Therefore, heterogeneity of proliferative activity is a crucial factor in the accurate estimation of tumor grade. Moreover, whether the LI or MI based on a single biopsy sample represents the entire tumor is uncertain, because the proliferative activity is not always homogeneous.

In this study, we aimed to characterize the heterogeneity of proliferative activity in gastric GISTs. For this purpose, we decided to examine the LI, because it is possible to obtain LI values for a large number of fields by using computer-assisted image analysis. In cases with LI heterogeneity, we determined the characteristic distribution of MIB-1-positive cells based on the tumor lobulation. 


\section{Patients and methods}

\section{Patients and pathological characteristics of ten GISTs}

Ten gastric GISTs showing c-kit positivity and with a diameter exceeding $3 \mathrm{~cm}$ were examined. Table 1 summarizes the patients' characteristics and the pathological features of the GISTs. All tumors were surgically resected at the Kyoto Prefectural University of Medicine, from 1997 through 2004. The patients were six men and four women, whose ages ranged from 33 to 79 years (median age, 56 years). Four patients had metastasis to the liver. The tumor sizes ranged from 30 to $100 \mathrm{~mm}$. The tumor growth pattern was endophytic in three cases, exophytic in four cases, and mixed endophytic and exophytic in three cases. All ten cases were spindlecell type. Immunohistochemically, all cases were positive for both c-kit and CD34.

\section{Immunostaining}

Immunohistochemical staining was performed with $5-\mu \mathrm{m}$-thick sections from formalin-fixed, paraffinembedded tissue. The sections included the maximum cut surface of the tumor mass. The sections were pretreated in an autoclave at $121^{\circ} \mathrm{C}$ for $10 \mathrm{~min}$ in $10 \mathrm{mmol} /$ 1 citrated buffer ( $\mathrm{pH}$ 6.0), and endogenous peroxidase activity was blocked with $0.3 \%$ hydrogen peroxide diluted in methanol for $30 \mathrm{~min}$. A standard indirect immunohistochemical procedure, using 3,3'diaminobenzidine as a chromogen, was performed with an anti-Ki-67 monoclonal antibody (clone MIB-1; Dakocytomation, Glostrup, Denmark). Counterstaining was carried out with Meyer's hematoxylin.

\section{Lobulation of GISTs}

While examining the hematoxylin-and-eosin-stained sections of the tumor, we distinguished multilobular GISTs (multiple lobules with intervening fibromuscular septa) from unilobular GISTs. Six tumors were multilobular, and four were unilobular (Table 2). The size did not differ significantly between multilobular tumors and unilobular tumors $(76.7 \pm 31.9 \mathrm{~mm}$ vs $57.5 \pm$ $23.6 \mathrm{~mm} ; P>0.1)$.

\section{Image analysis}

For each lobule, we obtained continuous fields of MIB1-immunostained sections extending through the maximum diameter of the cut surface, at $200 \times$ magnification. Figure 1 shows the gross appearance of a representative multilobular GIST (patient 1), with lines through which the fields of MIB-1-immunostained sections were obtained. These fields were digitized as JPEG images, using a digital camera (HC-300Zi; Nikon, Tokyo, Japan; Fig. 2A) and imported into a Power Macintosh G4

Table 1. Clinicopathological findings in ten patients with gastric GISTs

\begin{tabular}{|c|c|c|c|c|c|c|c|c|}
\hline $\begin{array}{l}\text { Patient } \\
\text { no. }\end{array}$ & $\begin{array}{c}\text { Age } \\
\text { (years) }\end{array}$ & Sex & Growth pattern & Metastasis & $\begin{array}{l}\text { Size } \\
(\mathrm{mm})\end{array}$ & c-kit & $\begin{array}{c}\text { CD34, SMA, } \\
\text { S100 }\end{array}$ & Lobulation \\
\hline 1 & 65 & M & Mixed & & 70 & + &,,+-- & + \\
\hline 2 & 65 & $\mathrm{M}$ & Mixed & (+) liver & 100 & + &,,+ \pm- & + \\
\hline 3 & 67 & $\mathrm{~F}$ & Mixed & & 100 & + &,,+ \pm- & + \\
\hline 4 & 77 & $\mathrm{M}$ & Endophytic & (+) liver & 60 & + &,,+-- & + \\
\hline 5 & 33 & $\mathrm{M}$ & Endophytic & (+) liver & 30 & + &,,+-- & + \\
\hline 6 & 64 & $\mathrm{~F}$ & Exophytic & & 100 & + &,,+-- & + \\
\hline 7 & 58 & $\mathrm{M}$ & Exophytic & (+) liver & 90 & + &,,+-- & - \\
\hline 8 & 51 & $\mathrm{M}$ & Endophytic & & 40 & + &,,+-- & - \\
\hline 9 & 79 & $\mathrm{~F}$ & Exophytic & & 60 & + &,,+-- & - \\
\hline 10 & 56 & $\mathrm{~F}$ & Exophytic & & 40 & + &,,+ \pm- & - \\
\hline
\end{tabular}

Table 2. Frequency of LI heterogeneity in multilobular and unilobular GISTs

\begin{tabular}{lccccc}
\hline & Lobulation & $n$ & MIB-1 LI heterogeneity & & $n$ \\
\hline GISTs $(n=10)$ & Multilobular & \multirow{2}{*}{6} & Intralobular & + & 6 \\
& & & Interlobular & + & 0 \\
& & & Intralobular & + & 1 \\
& & & & - & 3 \\
\hline
\end{tabular}




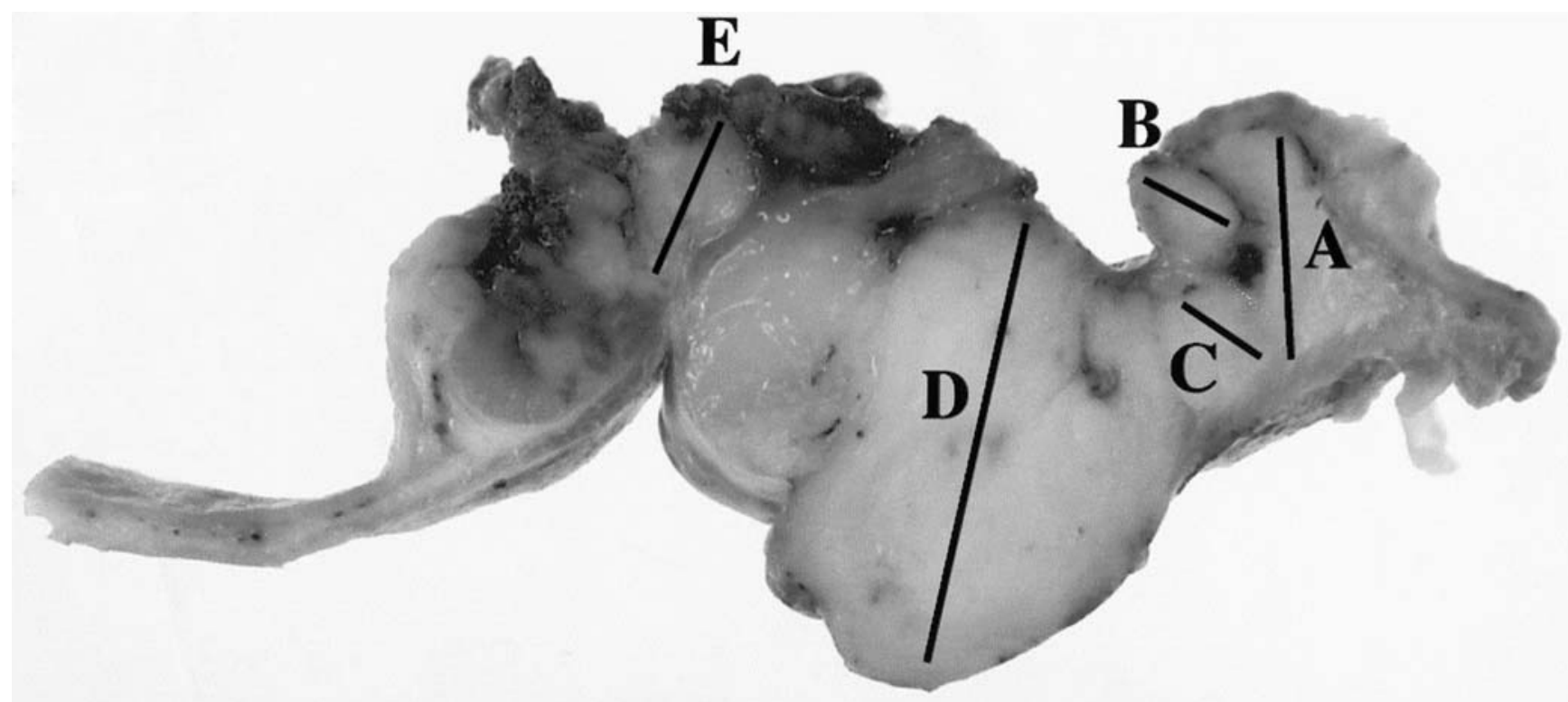

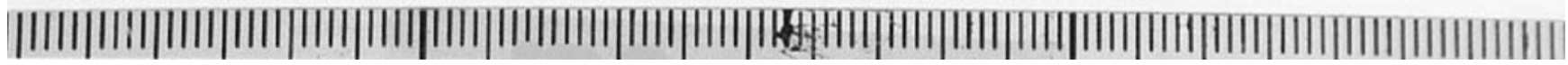

Fig. 1. Gross appearance of a multilobular gastrointestinal stromal tumor (GIST; case 1$)$. The lines $(A-E)$ indicate the maximum diameter of each lobule, through which continuous digital images of MIB-1-immunostained sections were obtained (see "Patients and methods")

computer (Apple Computers, Cupertino, CA, USA). The area of the MIB-1-stained nuclei (Fig. 2B) and the area of all nuclei, i.e., the nuclei stained by either MIB-1 or hematoxylin (Fig. 2C), were extracted in each digital image by the method of Hasegawa et al. [11], with some modifications. Then, the area ratio, which we designated as the computerassisted LI (CALI), was calculated with the following equation:

CALI $(\%)=100 \times$ MIB-1-positive area / (MIB-1positive area + hematoxylin-stained area)

The CALI is strongly correlated with the standard, microscopic observation-based LI. However, there may be section-to-section differences in the CALI, due to staining or thickness variability. Therefore, we adjusted the CALI values to allow comparison with standard LI values. Namely, from each lobule, we selected five images, including one with the highest CALI value and another with the lowest, and obtained standard LIs for these images with the following equation:

Standard LI $(\%)=100 \times$ number of MIB-1-positive nuclei / (number of MIB-1-positive nuclei + number of hematoxylin-stained nuclei)

With the CALI and standard LI values thus obtained, linear regression analysis was performed. The significance of the regression was judged by the regression slope with a $95 \%$ confidence interval. Then the CALI values, multiplied by the regression slope, were designated as the LI values and processed for further analysis.

\section{Evaluation of LI heterogeneity}

LI heterogeneity was divided into two categories according to the lobulation: intralobular (image-to-image manner) or interlobular (lobule-to-lobule manner). For each lobule, intralobular heterogeneity was evaluated on the basis of the SD. For multilobular tumors, interlobular heterogeneity was assessed on the basis of the mean LI difference between lobules.

\section{Results}

Table 3 summarizes the statistical values for the LI, tabulated in a lobule-by-lobule manner.

\section{LI heterogeneity in unilobular GISTs}

Unilobular GISTs showed mean LIs of $1.8 \%$ to $8.0 \%$, and SDs of $0.8 \%$ to $3.0 \%$ (Table 3 ). With an arbitrary definition of intralobular heterogeneity being present when the SD was greater than $2.5 \%$, only one of the four unilobular GISTs (cases 7 through 10) exhibited intralobular heterogeneity (Tables 2 and 3; Fig. 3). 

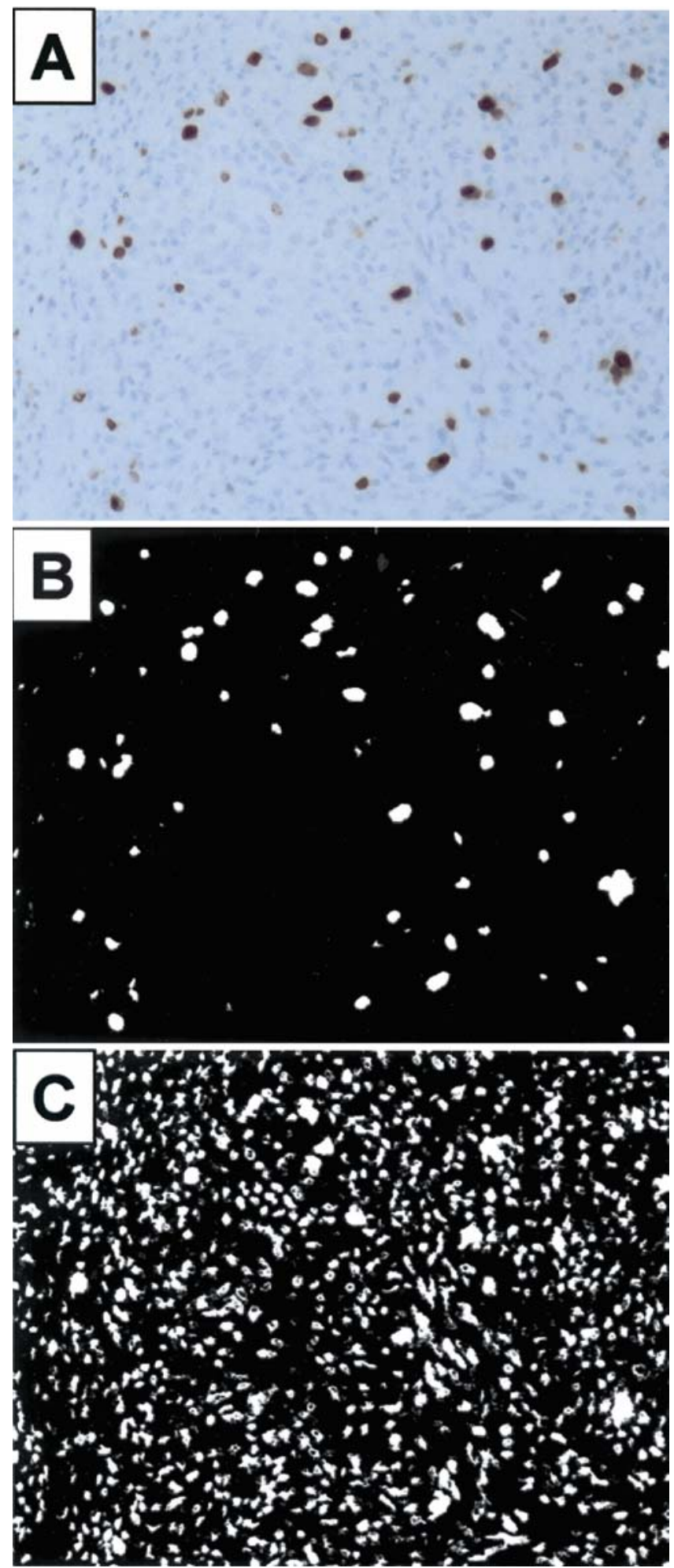

Fig. 2A-C. Image analysis. For the MIB-1-immunostained section (A), the area of the MIB-1-stained nuclei (B), and the area of all nuclei, i.e., the nuclei stained by either MIB-1 or hematoxylin $(\mathbf{C})$, were extracted by the method of Hasegawa et al. [11], with some modifications
Table 3. Statistical values for LIs of GISTs, tabulated in a lobule-by-lobule manner

\begin{tabular}{lcrrrrrr}
\hline $\begin{array}{l}\text { Case } \\
\text { no. }\end{array}$ & Lobule & $\begin{array}{r}\text { No. of } \\
\text { images }\end{array}$ & $\begin{array}{c}\text { Mean } \\
(\%)\end{array}$ & $\begin{array}{c}\text { SD } \\
(\%)\end{array}$ & $\begin{array}{r}\text { CV } \\
(\%)\end{array}$ & $\begin{array}{r}\text { Max } \\
(\%)\end{array}$ & $\begin{array}{r}\text { Min } \\
(\%)\end{array}$ \\
\hline 1 & A & 33 & 5.1 & 5.0 & 98 & 19.9 & 0.8 \\
& B & 21 & 32.3 & 9.8 & 30 & 46.3 & 17.9 \\
& C & 10 & 12.4 & 4.1 & 33 & 20.6 & 6.0 \\
& D & 88 & 7.0 & 5.0 & 71 & 23.0 & 0.2 \\
& E & 38 & 16.0 & 4.5 & 28 & 26.9 & 4.9 \\
2 & A & 54 & 26.9 & 7.2 & 27 & 41.7 & 6.1 \\
& B & 24 & 23.0 & 9.2 & 40 & 39.2 & 6.0 \\
& C & 40 & 4.6 & 3.2 & 70 & 13.9 & 0.3 \\
& D & 48 & 22.7 & 9.4 & 41 & 40.6 & 4.4 \\
& E & 73 & 21.8 & 4.5 & 21 & 35.6 & 12.7 \\
3 & A & 49 & 16.2 & 4.6 & 28 & 24.2 & 5.7 \\
& B & 30 & 2.7 & 1.0 & 39 & 5.0 & 0.8 \\
& C & 70 & 5.4 & 3.0 & 56 & 11.8 & 0.8 \\
4 & A & 65 & 27.7 & 5.5 & 20 & 39.1 & 14.7 \\
& B & 11 & 3.6 & 3.1 & 86 & 8.3 & 0.3 \\
5 & A & 33 & 14.1 & 4.2 & 30 & 24.5 & 4.5 \\
& B & 5 & 4.5 & 1.1 & 23 & 5.6 & 3.4 \\
6 & A & 46 & 4.8 & 2.6 & 54 & 12.5 & 1.7 \\
& B & 34 & 3.3 & 1.5 & 46 & 7.1 & 0.9 \\
7 & & 57 & 8.0 & 3.0 & 37 & 42.6 & 0.5 \\
8 & & 30 & 2.9 & 0.8 & 27 & 4.3 & 1.4 \\
9 & & 25 & 2.4 & 1.3 & 55 & 5.1 & 0.6 \\
10 & & 37 & 1.8 & 0.9 & 48 & 4.4 & 0.7 \\
\hline & & & & & & &
\end{tabular}

$\mathrm{CV}$, coefficient of variation

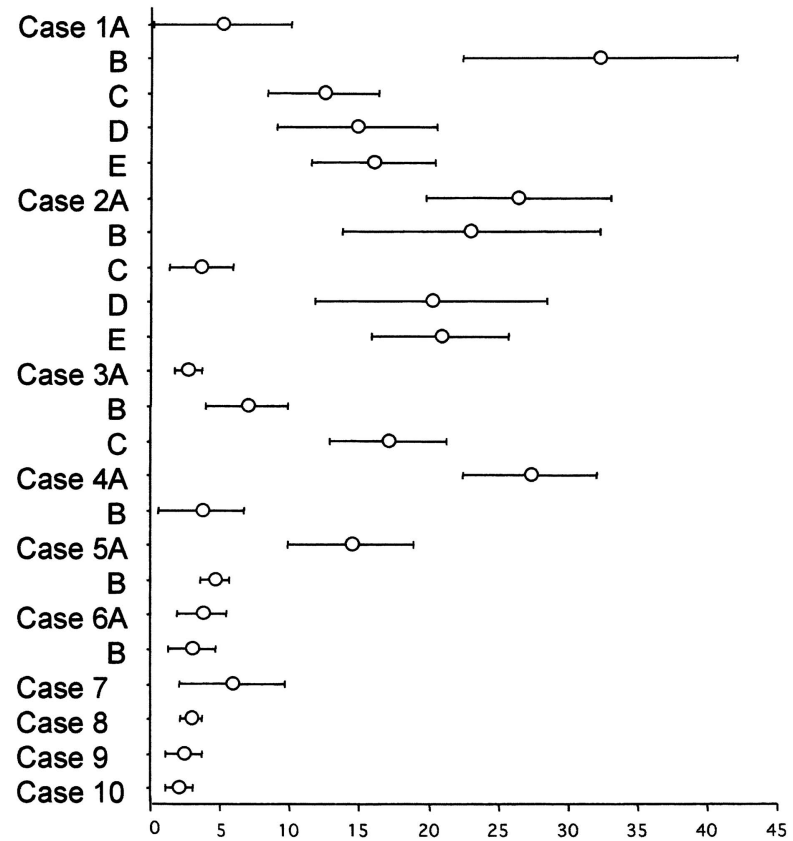

(\%)

Fig. 3. Labelling index (LI; mean $\pm \mathrm{SD}$ ) values for lobules in ten GISTs. All multilobular GISTs (cases 1 through 6) had at least one lobule with intralobular LI heterogeneity. Moreover, considerable interlobular heterogeneity was noted in five of the six multilobular GISTs (cases 1 through 5), with the maximum interlobular mean LI difference being $9.6 \%$ to $27.2 \%$. Unilobular GISTs (cases 7 through 10 ) tended to show homogeneous LI values, with SDs being $0.8 \%$ to $3.0 \%$ 


\section{LI heterogeneity in multilobular GISTs}

\section{Intralobular LI heterogeneity}

All multilobular GISTs had at least one lobule with intralobular LI heterogeneity. There was a significant correlation between the mean LI and SD (correlation coefficient, 0.86), showing that the greater was the mean LI, the greater was the intralobular heterogeneity (Table 3 and Fig. 3).

\section{Interlobular LI heterogeneity}

Considerable interlobular heterogeneity was noted in five of the six multilobular GISTs (cases 1 through 5), in which the maximum interlobular mean LI difference ranged from $9.6 \%$ to $27.2 \%$ (Table 3 ). On the other hand, interlobular LI heterogeneity was negligible for one multilobular GIST (case 6), because the mean LI difference was as low as $1.5 \%$.

The localization pattern of the lobules with high and low LIs was variable in the five multilobular GISTs with interlobular LI heterogeneity: in one GIST with an ulcer (case 1), the lobule with the highest LI (lobule 1B) was located at the edge of the ulcer; in another tumor (case 3), the lobule with the highest LI (lobule 3C) was located at the periphery of the tumor. On the other hand, in the other three tumors, relatively small lobules showed lower LIs than the rest of the tumor: in one tumor with a central cystic cavity (case 2), the lobule with low LI was located at the superior rim of the cyst (lobule 2C); in two other tumors (cases 4 and 5), minute lobules with low LIs (lobules 4B and 5B) were located at the periphery of the tumors.

All five cases with interlobular LI heterogeneity were considered high-grade tumors, considering that the maximum mean LI was $14.1 \%$ to $32.3 \%$. Notably, these tumors also contained at least one "low-grade" lobule, with a mean LI of $2.7 \%$ to $5.1 \%$.

Three multilobular GISTs with interlobular heterogeneity (cases 1, 4, and 5) had metastasized to the liver, whereas three unilobular tumors with a homogenous LI (cases 8 through 10) had not.

\section{Discussion}

This study has demonstrated, for the first time, that GISTs frequently show intratumoral LI heterogeneity. We classified intratumoral LI heterogeneity as intralobular or interlobular. Of these categories, interlobular LI heterogeneity was the key finding in this study. The mean LI of lobules was so divergent that some tumors could be considered a mixture of "high-grade" and "low-grade" lobules (Table 3; Fig. 3). Such heterogeneity may pose a problem in the examination of a single biopsy specimen of GIST, because a "low-grade" LI does not necessarily rule out the presence of "highgrade" lobules in the rest of the tumor. On the other hand, $60 \%$ (3 of 5) of the GISTs with interlobular heterogeneity, but none of the unilobular GISTs with homogeneous LIs, had metastasized to the liver. This finding suggests a possible relationship between interlobular LI heterogeneity and tumor malignancy.

There have been several reports of the intratumoral heterogeneity of proliferation markers in tumors other than GISTs. Kressner et al. [12] have shown that proliferative activity is heterogeneous in multiple biopsy samples of colorectal cancers, using three different markers, i.e., the MIB-1 labelling index, bromodeoxyuridine (BrdU) incorporation, and the DNA Sphase fraction. However, these authors did not specify the lobulation of the tumors. Interestingly, the intralobular heterogeneity in our GISTs was greater than that in the colorectal cancers in the study by Kressner et al. [12], shown by the coefficient of variation of the LI in our GIST lobules being $20 \%$ to $98 \%$ (mean, $44 \%$ ), whereas it was $4 \%$ to $61 \%$ (mean, $23 \%$ ) in the colorectal cancers. The difference is even greater if interlobular heterogeneity is taken into account.

Tumor progression is associated with intratumoral heterogeneity of genotype or phenotype, or both, in various tumors, which may lead to heterogeneous proliferative activity. For example, when Losi et al. [13] examined the evolution of intratumoral genetic heterogeneity during colorectal cancer progression, using $\mathrm{K}$-ras and p53 mutations and loss of heterozygosity as indicators, intratumoral genetic heterogeneity was clearly shown in early cancers, and it had decreased in advanced cancers and metastatic lesions. On the other hand, Matsuno et al. [14] have reported that the inner nodules of small hepatocellular carcinomas exhibit higher proliferating cell nuclear antigen LIs than do areas surrounding the tumor, indicating subclonal expansion within pre-existing tumors. For GISTs, the pathway of genetic changes has been determined in several cytogenetic studies, starting from c-kit mutation to successive chromosomal alterations $[15,16]$. However, intratumoral genetic heterogeneity in GISTs has not been documented, and, to our knowledge, this study is the first to report the heterogeneity of proliferative activity in GISTs, using the LI. Furthermore, considering the interlobular LI heterogeneity and the increased tendency for metastasis in multilobular GISTs shown in our study, it seems that clonal evolution may underlie the genetic progression and enhanced aggressiveness of GISTs. Studies to test this hypothesis are underway.

We examined GISTs of clinical size, i.e., those measuring more than $3 \mathrm{~cm}$ in diameter, but the degree and distribution of proliferative activity during early oncogenesis are unclear. Kato et al. [17] examined the concentration of MIB-1-positive cells in small gastric 
GISTs (less than $1 \mathrm{~cm}$ in diameter) and showed that they had more growth potential than did smooth muscle tumors (MIB-1-positive cells per $1 \mathrm{~mm}^{2}, 13$ to 24 vs 0.3 to 1.9). Surprisingly, this figure for small GISTs is much lower than the number of MIB-1-positive cells in our study, which ranged from 40 to 3000 per $1 \mathrm{~mm}^{2}$ (data not shown). These findings suggest that gastric GISTs show relatively high levels of proliferative activity from the early stage of development and, with growth, proliferative activity is continuously increased. Upon reaching clinical size, gastric GISTs also acquire a predilection for heterogeneous proliferative activity.

In conclusion, gastric GISTs of clinical size (larger than $3 \mathrm{~cm}$ in diameter) tend to show heterogeneous distribution of LIs, especially in a lobule-to-lobule manner. For predicting the behavior of gastric GISTs, consideration of this feature may be beneficial. For the preoperative evaluation of gastric GISTs, imaging studies, such as endoscopic ultrasonography, should be done to determine whether the tumor is multilobular. Because multilobular tumors tend to show interlobular LI heterogeneity, multiple biopsy samples may be necessary to detect the presence of high-grade lobules.

Acknowledgments The authors thank Mr. Toshifumi Kawamura and Mr. Takashi Okuda for technical assistance.

\section{References}

1. Fletcher CDM, Berman JJ, Corless C, Gorstein F, Lasota J, Longley BJ, et al. Diagnosis of gastrointestinal stromal tumors: a consensus approach. Hum Pathol 2002;33:459-65.

2. Miettinen M, El-Rifai W, Sobin LH, Lasota J. Evaluation of malignancy and prognosis of gastrointestinal stromal tumors: a review. Hum Pathol 2002:33;478-83.

3. Miettinen M, Sobin LH, Lasota J. Gastrointestinal stromal tumors of the stomach. a clinicopathologic, immunohistochemical, and molecular genetic study of 1765 cases with long-term followup. Am J Surg Pathol 2005;29:52-68.

4. Fujimoto Y, Nakanishi Y, Yoshimura K, Shimoda T. Clinicopathologic study of primary malignant gastrointestinal stromal tumor of the stomach, with special reference to prognostic factors: analysis of results in 140 surgically resected patients. Gastric Cancer 2003;6:39-48.

5. Hasegawa T, Matsuno Y, Shimoda T, Hirohashi S. Gastrointestinal stromal tumor: consistent CD117 immunostaining for diagnosis and prognostic classification based on tumor size and MIB-1 grade. Hum Pathol 2002;33:669-76.

6. Hasegawa T, Yamamoto S, Yokoyama R, Umeda T, Matsuno Y, Hirohashi S. Prognostic significance of grading and staging systems using MIB-1 score in adult patients with soft tissue sarcoma of the extremities and trunk. Cancer 2002;95:843-51.

7. Wong NACS, Young R, Malcomson RDG, Nayar AG, Jamieson LA, Save VE. Prognostic indicators for gastrointestinal stromal tumours: a clinicopathological and immunohistochemical study of 108 resected cases of the stomach. Histopathology 2003;43:11826.

8. Yan H, Marchettini M, Acherman YIZ, Gething SA, Brun E, Sugarbaker PH. Prognostic assessment of gastrointestinal stromal tumor. Am J Clin Oncol 2003;2:221-8.

9. Brown DC, Gatter KC. Ki67 protein: the immaculate deception? Histopathology 2002;40:2-11.

10. Seidal T, Edvardsson H. Expression of c-kit (CD117) and Ki67 provides information about the possible cell of origin and clinical course of gastrointestinal stromal tumours. Histopathology 1999; $34: 416-24$.

11. Hasegawa T, Yamamoto S, Matsuno Y. Quantitative immunohistochemical evaluation of MIB-1 labeling index in adult soft-tissue sarcomas by computer-assisted image analysis. Pathol Int 2002;52: 433-7.

12. Kressner U, Lindmark G, Gerdin B, Pahlman L, Glimelius B. Heterogeneity in proliferation markers in colorectal cancer. Anticancer Res 1995;15:2755-62.

13. Losi L, Baisse B, Bouzourene H, Benhattar J. Evolution of intratumoral genetic heterogeneity during colorectal cancer progression. Carcinogenesis 2005;26:916-22.

14. Matsuno Y, Hirohashi S, Furuya S, Sakamoto M, Mukai K, Shimosato Y. Heterogeneity of proliferative activity in nodule-innodule lesions of small hepatocellular carcinoma. Jpn J Cancer Res 1990;81:1137-40.

15. Corless CL, Fletcher JA, Heinrich MC. Biology of gastrointestinal stromal tumors. J Clin Oncol 2004;22:3813-25.

16. Heinrich MC, Rubin BP, Longley BJ, Fletcher JA. Biology and genetic aspects of gastrointestinal stromal tumors: kit activation and cytogenetic alterations. Hum Pathol 2002;33:48495.

17. Kato Y, Yamamoto N, Yanagisawa A. Pathology of gastrointestinal stromal tumor (GIST) with special reference to tumors in stomach. In: Kitamura Y, Miettinenn M, Hirota S, Kanakura Y, editors. Gastrointestinal stromal tumor (GIST): from pathology to molecular target therapy. Tokyo: Japan Scientific Societies Press; 2004. p. 161-75. 